\title{
Prospects for asteroid mass determination from close encounters between asteroids: ESA's Gaia space mission and beyond
}

\author{
Anatoliy Ivantsov ${ }^{1}$, Daniel Hestroffer ${ }^{2}$ and Siegfried Eggl ${ }^{2}$ \\ ${ }^{1}$ Fen Fakültesi, Akdeniz Üniversitesi, \\ Dumlupınar Bulvarı Kampüs, TR-07058, Antalya, Turkey \\ email: ivantsov@akdeniz.edu.tr \\ ${ }^{2}$ Institut de Mécanique Céleste et de Calcul des Éphémérides, Observatoire de Paris, \\ 77 avenue Denfert-Rochereau, F-75014 Paris, France
}

\begin{abstract}
We present a catalog of potential candidates for asteroid mass determination based on mutual close encounters of numbered asteroids with massive perturbers ( $D>20 \mathrm{~km}$ ). Using a novel geometric approach tuned to optimize observability, we predict optimal epochs for mass determination observations. In contrast to previous studies that often used simplified dynamical models, we have numerically propagated the trajectories of all numbered asteroids over the time interval from 2013 to 2023 using relativistic equations of motion including planetary perturbations, J2 of the Sun, the 16 major asteroid perturbers and the perturbations due to non-sphericities of the planets. We compiled a catalog of close encounters between asteroids where the observable perturbation of the sky plane trajectory is greater than 0.5 mas so that astrometric measurements of the perturbed asteroids in the Gaia data can be leveraged. The catalog v1.0 is available at ftp://dosya.akdeniz.edu.tr/ivantsov.
\end{abstract}

Keywords. Minor planets, asteroids, ephemerides, methods: numerical.

\section{Introduction}

Compared to the influence of the main Solar system bodies such as the Sun and the planets the gravitational interaction between asteroids tends to be small even during mutual close encounters. If the perturber is massive enough, close approach events can be used to constrain the mass of the perturbing asteroid through astrometric observation and analysis of the pre- and post-encounter trajectories. Given the large numbers of asteroids and potential mutual events the following questions become key to a successful asteroid mass determination campaign:

(a) Which asteroids should be observed?

(b) When should they be observed?

(c) What are the requirements to the accuracy of astrometric measurements allowing for reasonable constraints on a perturbing asteroid's mass?

In this paper we address the first two questions, as they can be answered to a satisfactory degree by precomputing asteroid-asteroid close encounters based on current trajectory information.

The 'classic' approach in modeling the close encounter between two asteroids has two evident assumptions: the gravitational interaction between the asteroids is instantaneous, so we can neglect interactions with other bodies during the close encounter, and the perturbed trajectory part of the small body can be considered a hyperbola with the centre of mass located at the nearest focus. The deflection of the velocity vector of the 
smaller asteroid due to the interaction with the more massive body can be used as a measure of the resulting perturbation.

The classic approach yields a seemingly simple way to assess the perturbation under certain assumptions. However, it is difficult to time observations in order to derive the precise asymptotes of the deflection hyperbola directly, given the number and magnitude of other gravitational interactions occurring at the same time. Close encounters between asteroids in the main belt are rather frequent, more than $1900 \mathrm{day}^{-1}$, with respect to the orbital revolution periods, see Sect. 3 . Also, the perturbing bodies are not necessarily located in the orbital plane of the perturbed asteroid, so the superposition of the perturbations will change the flat second-order curve to a more complex three-dimensional curve, in particular when encounters are slow. Hence, a more sophisticated model than the simple two body scattering approach is needed in order to determine when to best observe close encounters so as to maximise the encounter signal and minimize the noise due to other gravitational perturbations.

\section{Geometric approach}

Assuming the three-dimensional perturbed trajectory of an asteroid to be a regular curve, it is possible to introduce the Frenet-Serret frame fixed to each point of the trajectory. Here the orthonormal basis spanning the Euclidean space $\mathbb{R}^{3}$ is given by tangent, normal and binormal vectors. Using this frame any regular three-dimensional trajectory can be decomposed uniquely into two functions: curvature and torsion.

The curvature and torsion have local extrema with a clear meaning: minima are related to the most 'straight' or 'flat' elements of the trajectory with respect to the local neighbourhood while maxima give 'turning' points of the trajectory, a vertex, where the tangent or normal vectors experience the highest rate of change. In other words the minima of curvature and torsion correspond to the less perturbed elements of the trajectory while the maxima give the opposite case. The second order curves that are solutions of a two-body problem have the curvature $\kappa=\frac{|1+e \cos v|^{3}}{p\left(1+2 e \cos v+e^{2}\right)^{3 / 2}}$ and torsion $\tau=0$ where $p$ is the semi-latus rectum, $e$ is the eccentricity, and $v$ is the true anomaly of the perturbed orbit. As an example the curvature functions of (1) Ceres are presented in Fig. 1.

The trajectory of (1) Ceres resulting from a numerical integration of the equations of motion - the dynamical model is described in the following section - yields a large number of torsion and curvature extrema, Fig. 2. Those are a consequence of the perturbations by the planets and other asteroid perturbers, which are not in the same plane and change with time. All curvature and torsion functions of solar system objects will contain such contributions. We suggest to observe the perturbed asteroids at those epochs which coincide with the extrema of curvature and torsion closest to the encounter with the perturbing asteroid. The consecutive minima yield measurements of the greatest deflection angle or the 'accumulated effect' of perturbation while the intermediate maximum restricts the plane of the perturbation arc where the deflection angle is measured. An observational strategy based on this approach is bound to measure the greatest effect the perturber has on the perturbed body, before other perturbations change the orbit of the latter significantly.

\section{Algorithm and dynamical model}

In order to find the optimum epochs for observing the trajectory of perturbed asteroids we performed an exhaustive search of close encounters between 


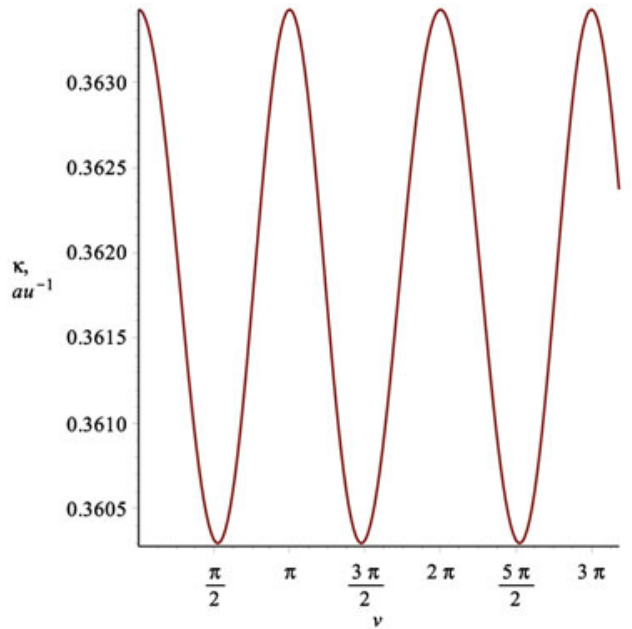

(a)

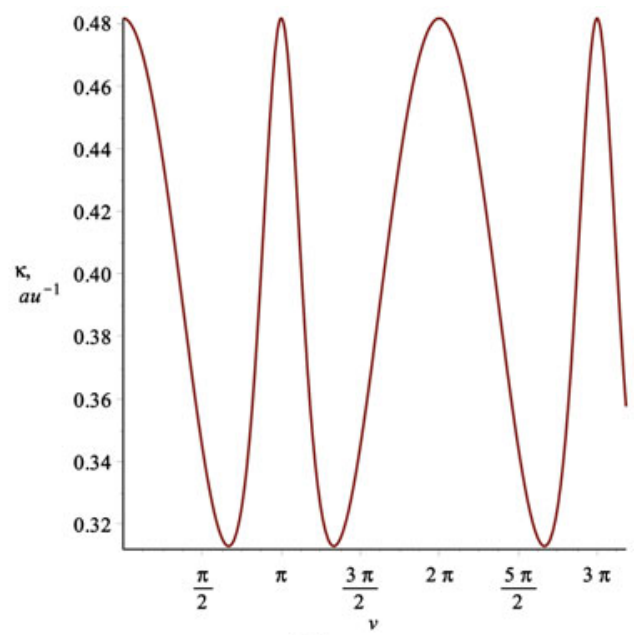

(b)

Figure 1. Curvature $\kappa(v)$ of the ideal orbit of (1) Ceres resulted from the two-body problem, $e=0.0757$ (a), and the same orbit with exaggerated eccentricity, $e=0.5$ (b).

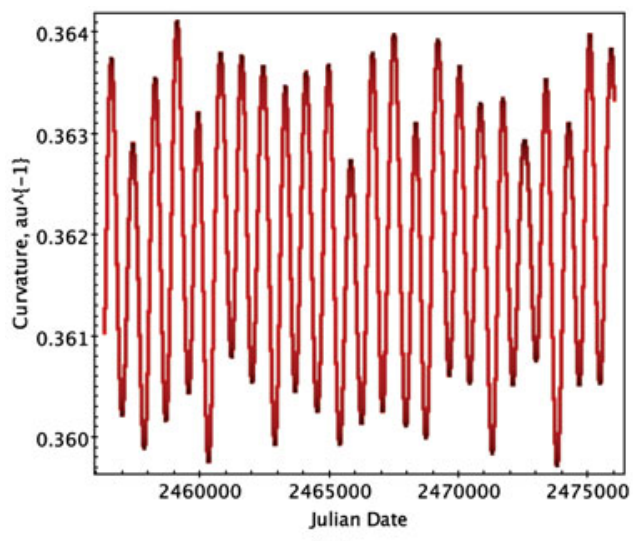

(a)

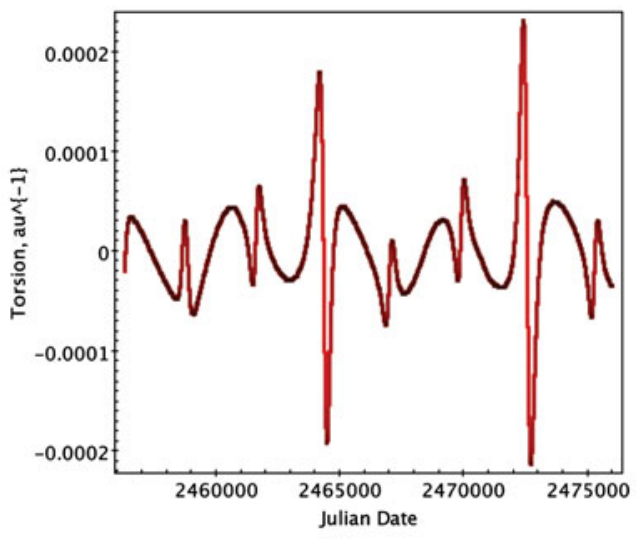

(b)

Figure 2. Curvature (a) and torsion (b) as functions of time resulting from an orbit-propagation of (1) Ceres.

$2191 \dagger$ massive asteroids and 488,449 numbered asteroids (and TNOs) within 2013-2023. To this end, we numerically propagated the equations of motion for each asteroid including all planetary perturbations and expanded the trajectories in series of Chebyshev polynomials. We then determined all local minima in the pairwise distances between asteroids and retained only those encounters which occurred at distances less than 0.1 au (6,941,633 encounters), see Table 1. After a curvature and torsion analysis, we selected only those encounters that yield skyplane deviations in the motion of perturbed asteroids greater than 0.5 mas $(535,182$ encounters). The complete dynamical model used to find the optimum observation epochs consists of Parametrized Post Newtonian equations of motion including the planetary ephemeris JPL DE431, perturbations from the

$\dagger$ Masses of the largest asteroids were found either from the publications or were calculated assuming equivalent spheres using the diameters measured by the IRAS (Tedesco et al. 2004) and the NEOWISE teams (Mainzer et al. 2011) and a density $3 \mathrm{~g} / \mathrm{cm}^{3}$. 
Table 1. Statistics of extreme values for the close encounters between asteroids at distances less 0.1 au between 2191 massive asteroids $(D>20 \mathrm{~km})$ and 488,449 numbered asteroids from 2013 till 2023 (total number of such encounters found is 6,941,633).

\begin{tabular}{|c|c|c|}
\hline Parameter & Minimum value & Maximum value \\
\hline Minimal distance, au & $2.34 \times 10^{-5}$ & 0.1 \\
Relative speed, au/day & $5.57 \times 10^{-6}$ & 0.0187 \\
Minimal distance in Hill's radii & 0.198 & 4397 \\
Deflection angle, arcsec & $0+$ & 249 \\
\hline
\end{tabular}

'BIG-16' asteroids (JPL HORIZONS), $J_{2}$ form factor of the Sun and non-sphericities of the planets to $J_{4}$. The propagator is based on an Adams-Moulton algorithm of variable order (1-12) and variable step; local relative error control is monitored to guarantee a relative error below $10^{-15}$ per step. Osculating elements of the processed asteroid orbits are based on data from JPL HORIZONS. Table 1 contains statistical information on the close encounter analysis.

\section{Discussion}

Curvature and torsion are invariant with respect to translations or rotations of the reference frame, however, both functions depend on the velocity and higher derivatives. The choice of the heliocentric or geocentric reference systems centered at the Sun or the Earth will introduce undesirable additional kinematic effects of the corresponding reference frames and should, thus, be avoided. This is the reason why the barycentric reference frame was used for the present calculations. The gravitational interaction between asteroids can be of the same order as Yarkovsky effect. The list of close encounters can furthermore be used for checking the adequacy of different ensembles of asteroids used in dynamical modeling.

\section{Conclusions}

We have presented a novel approach to determining optimum observation epochs for asteroid mass determination using asteroid-asteroid close encounters. The suggested approach for choosing observational moments corresponding to extrema in curvature and torsion of the perturbed asteroid trajectory can be useful for studying any particular orbit altering event, even if the target is subject to numerous other perturbations. Although the astrometric accuracy of the observations by the Gaia satellite is expected to be superior to the astrometry of the ground-based facilities, Gaia is not able to observe asteroids at specific epochs. Using the improved orbits resulting from Gaia data as well as the Gaia stellar catalog, close encounters between asteroids not to be observed by Gaia can be monitored by ground-based facilities. We have prepared a catalog listing excellent occasions and targets for astrometric observations that can greatly contribute to the effort of asteroid mass determination. The database of the close encounters and the observational moments is open to the public access. It is currently available at ftp://dosya.akdeniz.edu.tr/ivantsov. The interested reader is kindly requested to contact the corresponding author for further details.

\section{References}

Mainzer, A., Bauer, J., Grav, T., Masiero, J., Cutri, R. M., Dailey, J., Eisenhardt, P., McMillan, R. S., Wright, E., Walker, R., Jedicke, R., Spahr, T., Tholen, D., Alles, R., Beck, R., 
Brandenburg, H., Conrow, T., Evans, T., Fowler, J., Jarrett, T., Marsh, K., Masci, F., McCallon, H., Wheelock, S., Wittman, M., Wyatt, P., DeBaun, E., Elliott, G., Elsbury, D., Gautier, IV, T., Gomillion, S., Leisawitz, D., Maleszewski, C., Micheli, M., \& Wilkins, A. 2011, ApJ, 731, 53

Tedesco, E. F., Noah, P. V., Noah, M., \& Price, S. D. 2004, NASA Planetary Data System, IRAS Minor Planet Survey, IRAS-A-FPA-3-RDR-IMPS-V6.0 